ORIGINAL ARTICLE

\title{
Impact of the Department of Health initiative to equip and train acute trusts to manage chemically contaminated casualties
}

\author{
M Al-Damouk, A Bleetman
}

Emerg Med J 2005;22:347-350. doi: 10.1136/emj.2004.018580

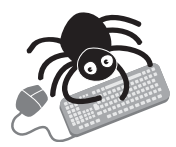

An appendix to this paper has been posted online at http://www.emjonline. com/supplemental/

See end of article for authors' affiliations

...................

Correspondence to: A Bleetman, Birmingham Heartlands Hospital, Bordesley Green East, Birmingham B9 5SS, UK; bleetman@enterprise.net

Accepted for publication 9 November 2004
Background: Before 1999, there was no national model or standard doctrine for managing casualties from chemical incidents in the UK. A Department of Health (DoH) initiative to prepare the National Health Service (NHS) for chemical incidents was launched in the same year. This led to the distribution of an NHS standard chemical personal protective equipment suit (CPPE) together with a new single half day training package (Structured Approach to Chemical Casualties (SACC)) in 2001.

Objectives: To assess the impact of the DoH initiative on acute hospital and ambulance trusts. To identify deficiencies in the design and operational deployment of the new CPPE, training initiative, and decontamination procedures at hospital level.

Method: A survey to assess progress in specific areas of chemical incident preparedness and two simulated incidents with "live" chemically contaminated casualties conducted in two acute trusts. Umpires evaluated the operational performance against DoH SACC standards.

Results: There has been marked improvement in many aspects of preparedness for chemical incidents since the original National Focus survey. Some deficiencies remain and this study identified areas for further work. In the live casualty exercises, hospital staff complied well with SACC protocols. Some practical difficulties were encountered with the deployment of the CPPE and in some aspects of the operational response, leading to some delays in the delivery of care to the casualties and to the integrity of the uncontaminated (clean) zones within the hospitals.

Conclusion: Problems with the design and deployment of the CPPE, together with training difficulties have been fed back into the planning and development process.
A survey of hospitals conducted by the National Focus for Chemical Incidents in 1999/2000 demonstrated that acute trusts were largely unprepared to deal with chemical incidents. ${ }^{1}$ In 2001, a Department of Health initiative conducted by a Delphi process led to the provision of chemical personal protection equipment (CPPE) to both acute hospital and ambulance trusts. ${ }^{12}$ The CPPE comprised the "NHS suit" and an inflatable decontamination shelter. The equipment was accompanied by the Structured Approach to Chemical Casualties (SACC) training package. ${ }^{3}$

Following the terrorist attacks on the World Trade Center in September 2001, the fear of terrorist activity in the UK (including the use of chemical weapons) precipitated the provision of 2500 interim sets of CPPE to the health service in high density population areas. In November 2002 an improved final version of the CPPE was distributed together with the SACC training package to all acute hospital and ambulance trusts. ${ }^{3}$ The training package familiarises staff with the use of CPPE, individual patient decontamination, medical management of chemical casualties, and maintenance of scene safety. ${ }^{3}$

An assessment of the impact of this initiative will inform planners of the preparedness of acute trusts to respond to chemical incidents.

\section{METHODS}

We distributed a questionnaire to lead SACC trainers and department heads in all 224 UK acute hospital and ambulance trusts. We then conducted simulated chemical incidents using volunteer simulated casualties in two emergency departments in the West Midlands. Twelve children participated in the exercise at the regional children's hospital and eight fire fighters at an acute general hospital. Both participating hospitals had received the CPPE and had delivered SACC training to all Accident and Emergency (A\&E) staff.

We held a series of preparatory planning meetings at both hospitals to obtain consent, conduct risk assessments, and to determine the scope of the exercises. Observers from the emergency services were in attendance.

The volunteer casualties were made-up to simulate chemical contamination and associated physical blast injuries. An ultraviolet medium was applied to parts of their body to evaluate the efficacy of decontamination. A 20 minute warning of a simulated chemical incident was given to each department before the arrival of the simulated casualties. We deployed observers from the emergency services, a nurse, and two resuscitation officers to key areas inside and outside the departments to observe specific areas of staff response. The casualties were led across in small groups to the emergency department 20 minutes after the activating call. At both hospitals, most of the casualties were walking, one was on a stretcher, one was briefed to collapse at the triage point, and another was briefed to run into the A\&E looking for a friend. The exercises were terminated after the last casualty had been processed.

We followed each exercise with an interactive debriefing. The volunteers were debriefed separately. Compliance with SACC teaching and modern major incident planning guidance was assessed by the observers on pre-prepared marking

Abbreviations: A\&E, Accident and Emergency; CPPE, chemical personal protection equipment; SACC, Structured Approach to Chemical Casualties 
Table 1 Results of the questionnaire survey

\begin{tabular}{|c|c|c|}
\hline & Ambulance trust $(n=6)$ & Emergency departments $(n=49)$ \\
\hline \multicolumn{3}{|l|}{ General information } \\
\hline No of COMAH sites in area & Range $0-78$, average 20 & Range $0-36$, average 5 \\
\hline $\begin{array}{l}\text { No of chemical incidents dealt with in } \\
\text { previous } 12 \text { months }\end{array}$ & Range $0-371$ average 74 & Range $0-4$, average 1 \\
\hline \multicolumn{3}{|l|}{ Equipment and facilities } \\
\hline $\begin{array}{l}\text { Estimated time to set up shelter + make it } \\
\text { operational (minutes) }\end{array}$ & Mean 18.5 , median 15 , range $14-30$ minutes & Mean 19.86 , median 17.5 , range $2-60$ minutes \\
\hline CPPE & All 6 respondents (100\%) had CPPE & $48 / 49$ (98\%) had CPPE \\
\hline & $5 / 6(83 \%)$ had trained staff in its use & $40 / 49(82 \%)$ had trained staff in its use \\
\hline \multicolumn{3}{|l|}{ Contaminated effects and water disposal } \\
\hline Disposal of contaminated effects & $\begin{array}{l}4(67 \%) \text { had arrangements to deal with } \\
\text { contaminated clothing }\end{array}$ & $\begin{array}{l}2(4 \%) \text { had arrangements to deal with contaminated } \\
\text { clothing }\end{array}$ \\
\hline Water disposal & $\begin{array}{l}4(67 \%) \text { had storage tanks } \\
3(50 \%) \text { would use the public sewer }\end{array}$ & $\begin{array}{l}35(71 \%) \text { had a storage tank for water run-off } \\
10(20 \%) \text { would use the public sewer } \\
1(2 \%) \text { had a private drainage site } \\
1(2 \%) \text { used storage barrels }\end{array}$ \\
\hline Exercising in the previous 12 months & $\begin{array}{l}3(50 \%) \text { had conducted chemical exercises } \\
5(83 \%) \text { had plans to exercise within the next } \\
12 \text { months }\end{array}$ & $\begin{array}{l}17(34 \%) \text { had conducted chemical exercises } \\
35(71 \%) \text { had plans to exercise within the next } 12 \text { months }\end{array}$ \\
\hline
\end{tabular}

sheets (see appendix online at http://www.emjonline. com/supplemental/).

\section{RESULTS}

\section{Survey}

We sent questionnaires to 192 hospitals and 32 ambulance trusts in the UK. Of these, 49/192 hospitals (26\%) and 6/32 ambulance trusts responded (19\%). The responses are presented in table 1 .

\section{Simulated chemical incidents}

Compliance with SACC protocols from the two simulated chemical incidents at both hospitals is summarised in table 2 .

\section{Other observations from the exercises}

\section{Zone boundaries}

There was inadequate control of pedestrian flow across the zone boundaries in both hospitals.

\section{CPPE}

The CPPE suits were found to be very fiddly and cumbersome to assemble and took trained staff at least 15 minutes to prepare, test, and don. There were frequent battery failures. Two suits in one hospital failed due to battery malfunction after 15 minutes. Staff commented that the suits provided inadequate flexibility at the knees, shoulders, and elbows when lifting. The visor was pulled backwards by the weight of the internal air pump. The suits leaked in the foot area. Manual handling was considerably impaired.

\section{Inflatable shelter}

At both hospitals, the process of preparing and erecting the shelter took approximately four minutes. In the children's hospital chemical incident plan, it was the task of the estates personnel to erect the decontamination shelter to allow clinical staff to prepare for and receive casualties. At the general hospital, clinical staff were expected to perform both

Table 2 Assessment of staff performance and compliance with the SACC doctrine during the simulated chemical incident exercises

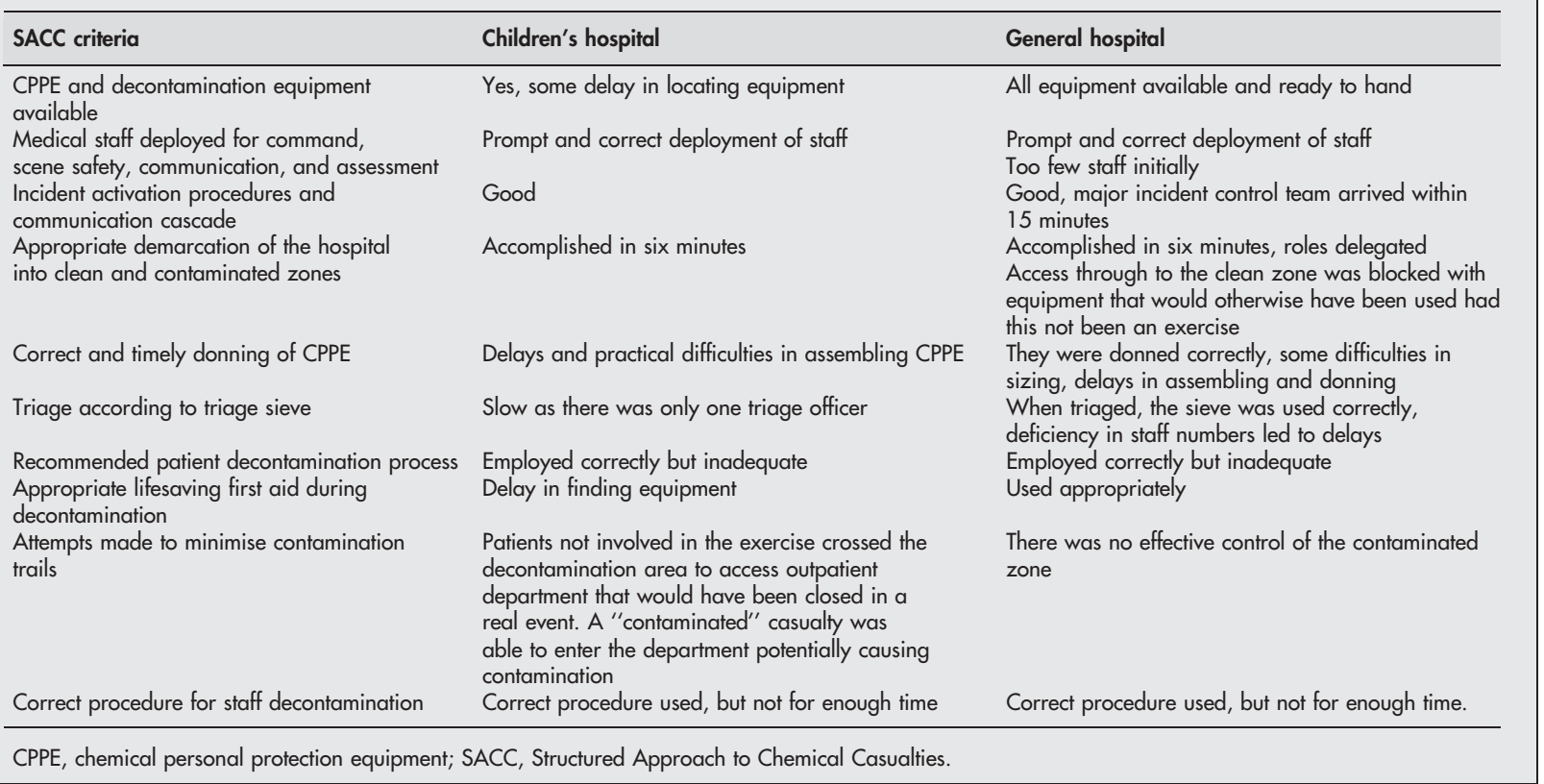




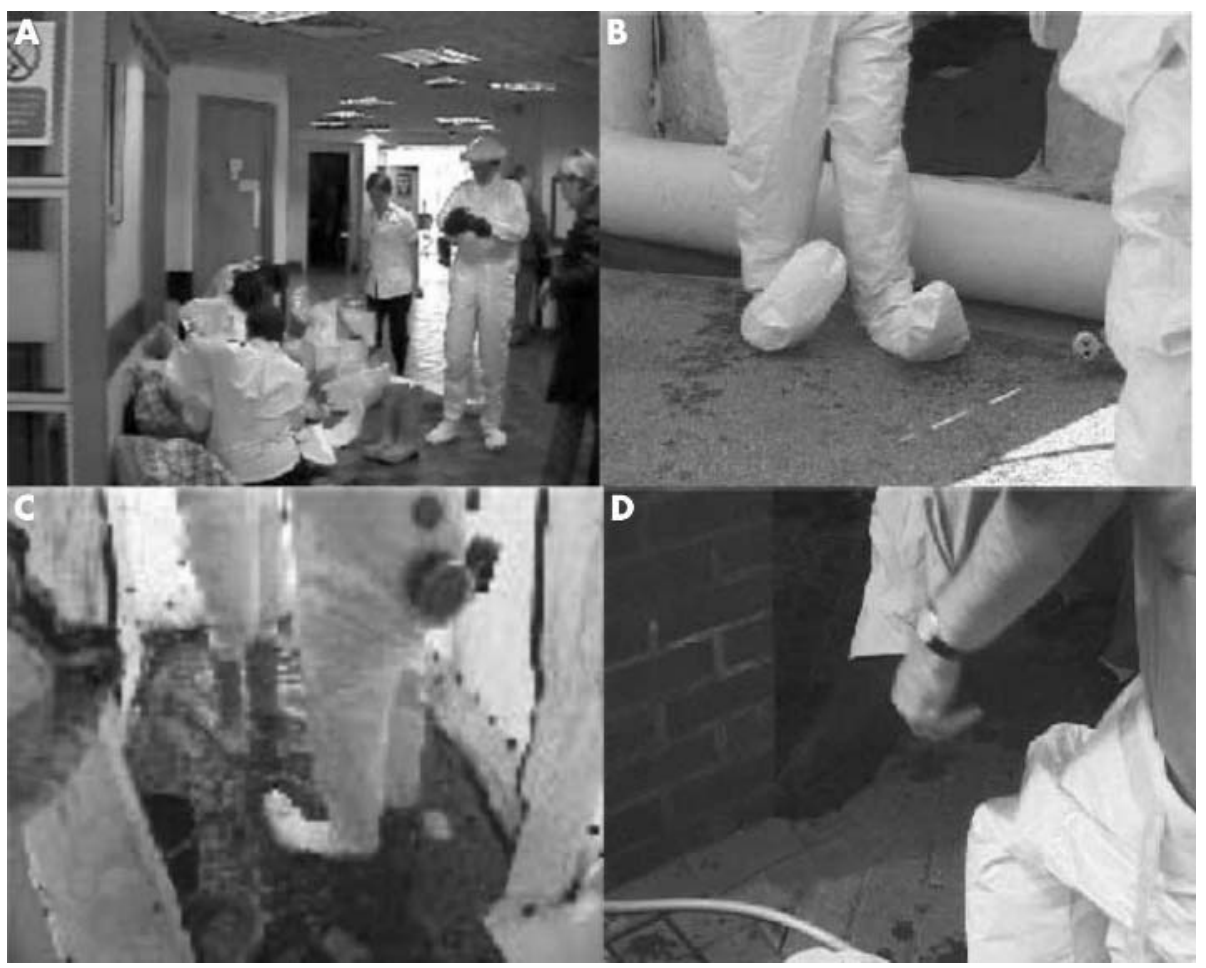

Figure 1 Difficulties encountered with the NHS suit. (A) Difficulties in donning the chemical personal protection equipment (CPPE). (B) Difficulties in sizing CPPE. (C) Weight distribution of battery and filter within CPPE. (D) Leakage of fluid into CPPE.

these tasks. This overstretched clinical staff and compromised their ability to assemble an adequate clinical response in the first few minutes of the exercise.

The water pressure remained poor throughout the exercise at the general hospital. One shelter was inflated fully but was then allowed to deflate because the non-return valve was not secured immediately. This was corrected after some difficulty. The water level in the shelter at the end of the exercise was high enough to overspill.

\section{Patient management}

There were manual handling difficulties as there was no trolley to move collapsed patients from the delivery point into the shelter and decontamination process. In both hospitals, staff failed to decontaminate patients adequately as demonstrated with the ultraviolet medium.

\section{After the incident}

Staff decontamination was generally poor. There was leakage in the bootee area of the new NHS suit. At both hospitals, no one was certain of the correct procedures for the disposal of the contaminated garments. The removed garments were left uncovered in the open.

\section{Hot debriefing observations from participants}

Staff struggled with preparing and donning the suit. Those who wore the CPPE found it difficult to hear others, even when both parties were shouting. Most felt uncomfortable in the suits after half an hour, even though most were in them for 90 minutes due to inadequate staff numbers to relieve them.

It was felt that it would be impossible to deploy staff rapidly and safely should chemical casualties arrive at hospital with little or no warning. Some of the difficulties in preparing and donning the CPPE are illustrated in fig 1.

\section{DISCUSSION}

The journey to provide the health service with a new comprehensive solution for chemical incidents began in
1999. Before that, there had been no consensus on how to approach the challenges of dealing with chemical incidents. Most units were woefully unprepared. A Department of Health led Delphi process delivered the dogma, training, process, and equipment for the management of these incidents to the health service. The intention was to prevent further contamination of casualties and staff, and also to provide a structured and common health service approach to contaminated casualty and scene management. The events of 11 September 2001 accelerated this process and the equipment and training package was delivered to the health service in November 2002. At the time of this study, most units had received their CPPE and training package, delivered by the regions. ${ }^{34}$

Ambulance services deal with chemical incidents far more frequently than acute hospital trusts. They are often summoned to attend chemical spills by the fire service as a precaution but are rarely required to transport chemically contaminated casualties to hospital (personal communication, West Midlands Ambulance Service). Hospitals, however, face a different risk. They are required to respond to chemical incidents only rarely. When they do, casualties will often self-present without prior notice and therefore staff must be able to respond very quickly.

The simulated chemical incidents conducted at two hospitals serves to demonstrate the reality of the challenge faced by staff responding to this type of incident. The user's ability to cope with the decontamination equipment, preparation and testing of the CPPE suit, and donning and doffing it safely under pressure is central and implicit in any coherent and timely response to a chemical incident. This is particularly the case for hospital staff who are likely to face the challenge of chemically contaminated casualties attending the hospital unannounced, or after minimal notice. This has been witnessed in many incidents worldwide; many will not wait for the emergency services to attend the scene of an accident and will make their own way to the nearest hospital. ${ }^{5-7}$ Hospital staff therefore need to be prepared to respond at very short or no notice to the challenges of a chemical incident. 
Although the training package and dogma seem to have been delivered effectively to hospitals, the current suit and inflatable shelter pose a number of considerable ergonomic and functional difficulties. The suit is prepacked, fiddly, and takes a long time to rig, test, and don. Further improvements are required (Department of Health Working Group, Mark II project (NHS CPPE), unpublished). The package deals exclusively with chemical incidents but has not addressed radiation or biological incidents. Clearly, a unified management and equipment package for health staff to manage chemical, biological, radiological, and nuclear (CBRN) incidents would be better than having a separate approach and equipment for each component of CBRN. Closer collaboration with other agencies is required.

Although the comparison of the data between the two surveys cannot be tested for statistical significance, it appears that there has been a trend towards improvement in several key areas. Trusts have more equipment than they did before; most now appear to have a specific chemical incident plan. Training too has been provided for more staff than ever before.

We would urge the designers of the next generation of CPPE to minimise the number of stages in preparing, testing, and donning equipment so that staff, who may only have had short exposure to it in training will be able to respond promptly, efficiently, and safely to the unexpected presentation of chemically contaminated casualties.

\section{ACKNOWLEDGEMENTS}

Professor K Mackway-Jones, Dr B Wilson, Dr I Crawford, West Midlands Fire Service, Edgbaston High School for Girls, National Focus for Chemical Incidents.

\section{Authors' affiliations}

M Al-Damouk, Sandwell General Hospital, Birmingham, UK

A Bleetman, Birmingham Heartlands Hospital, Bordesley Green East, Birmingham, UK

Competing interests: none declared

\section{REFERENCES}

1 Russell D, Mackway-Jones K, Crawford IWF. Draft paper: Chemical Incident Preparedness in Emergency Departments and Ambulance Services in England. Withdrawn from submission following the attack on the World Trade Centre.

2 Crawford IWF, Mackway-Jones K, Russel DR, et al. Planning for chemical incidents by implementing a Delphi based consensus study. Emerg Med J 2004;21:20-3.

3 ALSG Training Masters Revised, Structured Approach to Chemical Casualties (SACC). Adult Life Support Group. Manchester, November, 2001.

4 Murray V, Mackway-Jones K. Pre-Hospital Chemical, Biological Incident Response; Bleetman A, et al. A Briefing Document. NeLH 23 October 2001 5 Karsenty E, Shemer J, Alshech I, et al. Medical aspects of the Iraqi missile attacks on Israel. Isr J Med Sci 1991;27(11-12):603-7.

6 Nakajima T, Ohta S, Morita H, et al. Epidemiological study of sarin poisoning in Matsumoto city, Japan. J Epidemio 1998;8:33-41

7 Okumura T, Suzuki K, Fukuda A, et al. The Tokyo subway sarin attack: disaster management, part 1: community emergency response. Acad Emerg Med 1998:5:613-17. 